\title{
Correction to: A Communication Protocol for Decentralized Fault Diagnosis of Discrete Event Systems
}

\author{
Milad Khaleghi $^{1} \cdot$ Mojtaba Barkhordari Yazdi ${ }^{1}$ (D) Ali Karimoddini $^{2} \cdot$ Malihe Maghfoori Farsangi $^{1}$
}

Published online: 2 November 2021

(c) Shiraz University 2021

\section{Correction to: Iranian Journal of Science and Technology, \\ Transactions of Electrical Engineering \\ https://doi.org/10.1007/s40998-021-00457-2}

In this article the co-author's name and the email address

was incorrectly written as

M. M. Farsangi

m.farsangi@uk.ac.ir

It should have been

Malihe Maghfoori Farsangi

mmaghfoori@uk.ac.ir

The original article has been corrected.

The original article can be found online at https://

doi.org/10.1007/s40998-021-00457-2.

Mojtaba Barkhordari Yazdi

barkhordari@uk.ac.ir

Milad Khaleghi

khaleghi.milad@gmail.com

Ali Karimoddini

akarimod@ncat.edu

Malihe Maghfoori Farsangi

mmaghfoori@uk.ac.ir

1 Department of Electrical Engineering, Shahid Bahonar

University of Kerman, Kerman 76175-133, Iran

2 Department of Electrical and Computer Engineering, North

Carolina Agricultural and Technical State University,

Greensboro, NC, USA 